\title{
On the exploitation of chaos to build reduced-order models
}

\author{
G. Kerschen, J.-C. Golinval, \\ Département d'Aérospatiale, Mécanique et Matériaux, \\ Vibrations et Identification des Structures, \\ Université de Liège, \\ Chemin des Chevreuils 1 (B52), B-4000 Liège, Belgium. \\ B.F. Feeny, \\ Department of Mechanical Engineering, \\ Michigan State University, \\ Engineering Building, East Lansing, MI 48824, USA.
}

\begin{abstract}
The present study focuses on the model reduction of non-linear systems. The proper orthogonal decomposition is exploited to compute eigenmodes from time series of displacement. These eigenmodes, called the proper orthogonal modes, are optimal with respect to energy content and are used to build a low-dimensional model of the non-linear system. For this purpose, the proper orthogonal modes obtained from a chaotic orbit are considered. Indeed, such an orbit is assumed to cover the phase space more uniformly. It is shown that the modes for this particular behaviour are more representative of the system dynamics than any other set of modes extracted from a non-chaotic response. This is applied to a buckled beam with two permanent magnets and the reduced-order model is validated using both qualitative and quantitative comparisons.
\end{abstract}

\section{Introduction}

In many domains of applied sciences and in structural dynamics particularly, dealing with large-scale dynamical structures is a central issue. In the presence of non-linearities, seeking for the solution by use of mathematical modelling and simulation (e.g., finite element method) may be computationally intensive. Accordingly, due to the complexity of such a numerical approach, it is worth reducing the dimensionality of the system while retaining its intrinsic properties.

The general philosophy of model reduction is to find a co-ordinate transformation in order to sort the components in terms of their influence on the system behaviour. Then, the components of the transformed system with relatively small influence may be truncated without substantially degrading the predictive capability of the model. The proper orthogonal decomposition (POD), also known as Karhunen-Loève transform or principal component analysis, enables such a co-ordinate transformation. It is a statistical pattern analysis technique for finding the dominant structures in an ensemble of spatially distributed data. These structures, called the proper orthogonal modes (POMs), may be exploited as an orthogonal basis for efficient representation of the ensemble. A key advantage of the decomposition is that each POM is associated with a proper orthogonal value (POV) which provides the relative energy captured by the corresponding mode. Thus, it serves as a well-defined measure of a mode influence on the system behaviour.

The present study is motivated by the fact that, in the field of non-linear systems, new features must be defined because mode shapes are no longer effective to represent the system dynamics. While some similarities between the POMs and the modes shapes have been noticed [1,2], the POMs are much more useful for capturing the dynamics of a non-linear system. In reference [3], lower dimensional models of non-linear vibrating systems are created using the POMs in order to prove their efficiency and their superiority over the mode shapes.

In this work, the POMs obtained from a chaotic orbit are considered. Indeed, such an orbit is assumed to cover the phase space more thoroughly than a periodic orbit. It is shown in an example that the modes for this particular behaviour are more representative of the system dynamics than any other set of modes extracted from a non-chaotic response. This is applied to a buckled beam with two permanent mag- 
nets and the reduced-order model is validated using both qualitative and quantitative comparisons while the idea was also applied to frictionally excited systems [4].

\section{Proper orthogonal decomposition}

The POD, also known as the Karhunen-Loève decomposition, was proposed independently by several scientists including Karhunen [5], Kosambi [6], Loève [7], Obukhov [8] and Pougachev [9] (see reference [10] for a recent survey) and was originally conceived in the framework of continuous second-order processes. When restricted to a finite dimensional case and truncated after a few terms, the POD is equivalent to principal component analysis (PCA). This latter methodology originated with the work of Pearson [11] as a means of fitting planes by orthogonal least squares and was also proposed by Hotelling [12].

The first applications of the POD in the field of structural dynamics date back to the early nineties. Cusumano et al. [13] exploited the technique to estimate the intrinsic dimensionality of the dynamics of an impacting beam. Kreuzer and Kust [14] used it to control self-excited vibrations of long torsional strings. Kappagantu and Feeny [4] worked on the modal reduction of a frictionally excited system. Other studies include the works of Azeez and Vakakis [15], Benguedouar [16], Fitzsimons and Rui [17], Georgiou and Schwartz [18] and Lenaerts et al. [19].

The central idea of the POD is to reduce a large number of interdependent variables to a much smaller number of uncorrelated variables while retaining as much as possible of the variation in the original variables. An orthogonal transformation to the basis of the eigenvectors of the sample covariance matrix is performed and the data is projected onto the subspace spanned by the eigenvectors corresponding to the largest eigenvalues. This transformation decorrelates the signal components and maximises variance. The most striking property of the POD is its optimality in the sense that it minimises the average squared distance between the original signal and its reduced representation.

For the sake of brevity, the complete mathematical description of the POD is not recalled here. The reader is referred to reference [20] for a detailed description. For practical applications, the data is discretised in space and time. Accordingly, $m$ observa- tions of a $n$-dimensional vector $\mathbf{x}$ are collected and an $(n \times m)$ response matrix is formed:

$$
\mathbf{X}=\left[\begin{array}{lll}
\mathbf{x}_{1} & \cdots & \mathbf{x}_{m}
\end{array}\right]=\left[\begin{array}{ccc}
x_{11} & \cdots & x_{1 m} \\
\cdots & \cdots & \cdots \\
x_{n 1} & \cdots & x_{n m}
\end{array}\right]
$$

The POMs and POVs are defined as the eigenvectors and eigenvalues of the covariance matrix $\mathbf{~ = ~}$ $E\left[(\mathbf{x}-\boldsymbol{\mu})(\mathbf{x}-\boldsymbol{\mu})^{T}\right]$ respectively, where $E[\cdot]$ is the expectation and $\boldsymbol{\mu}=E[\mathbf{x}]$ is the mean of the vector $\mathbf{x}$. Under the assumption that the process is ergodic and that the number of observations is large, a reliable estimate of the covariance matrix is given by the sample covariance matrix. If the data is zero mean, the sample covariance is merely given by the following expression:

$$
\mathbf{S}=\frac{1}{m} \mathbf{X X}^{T}
$$

The POMs and POVs are characterised by the eigensolutions of the sample covariance matrix $\mathbf{S}$.

\section{Reduced-order models}

Conventionally, one of the methods used to analyse non-linear systems is through projection of the equations of motion onto the modes shapes of the linearised system. This method often requires the concurrent simulation of many modes to achieve a satisfactory accuracy, and the resulting model may be computationally expensive. The approach presented herein conjectures that a reduced-order model based on the POMs allow for much more efficient analysis of the original system. Furthermore, due to the 'random nature' of chaos, the POMs of a chaotic orbit are expected to better capture the system dynamics than any other set of POMs extracted from a non-chaotic response.

Even though the original physical model typically has an infinite number of degrees of freedom to start with, it is assumed that the equations of motion have already been discretised in space by using a finite element model. For a non-linear mechanical structure, the spatial discretisation usually yields the following equations:

$$
\mathbf{M} \ddot{\mathbf{x}}(t)+\mathbf{C} \dot{\mathbf{x}}(t)+\mathbf{K x}(t)+\mathbf{f}_{N L}(\mathbf{x}(t), \dot{\mathbf{x}}(t))=\mathbf{f}(t)
$$

where $\mathbf{M}, \mathbf{C}, \mathbf{K}$ are the mass, damping and stiffness matrices respectively; $\mathbf{x}$ is the vector of displacement co-ordinates; $\mathbf{f}_{N L}$ is the non-linear force vector; $\mathbf{f}$ is the external applied force vector. 
However, this may lead to a large number $n$ of degrees of freedom if a sufficient approximation of the true physical phenomenon is required. In order to reduce the complexity, model reduction techniques are to be considered. They may be viewed as a projection of the $n$-dimensional vector of displacement coordinates $\mathbf{x}(t)$ to a vector $\mathbf{a}(t)$ of lower dimension $r$. The co-ordinate transformation considered in this study is defined by an $(n \times r)$ projection matrix $\mathbf{P}$ whose columns are the POMs corresponding to the $r$ greatest POVs:

$$
\mathbf{x}(t)=\mathbf{P a}(t)+\boldsymbol{\mu}
$$

The equations of motion expressed in the lower dimensional setting are as below:

$$
\begin{aligned}
\ddot{\mathbf{a}}(t)+\mathbf{C}^{*} \mathbf{\mathbf { a }}(t)+\mathbf{K}^{*} \mathbf{a}(t)+(\mathbf{M P})^{\dagger} \mathbf{K} \boldsymbol{\mu}+ \\
(\mathbf{M P})^{\dagger} \mathbf{f}_{N L}=(\mathbf{M P})^{\dagger} \mathbf{f}(t)
\end{aligned}
$$

where $\mathbf{C}^{*}=(\mathbf{M P})^{\dagger} \mathbf{C P}, \mathbf{K}^{*}=(\mathbf{M P})^{\dagger} \mathbf{K P}$ and $\dagger$ is the pseudo-inverse. The solution in the original coordinate system can be retrieved using Eq. (4). This of course does not yield the correct solution but an approximation whose quality depends on the severity of the reduction.

It remains to determine the order of reduction, i.e., the number of POMs to be included in matrix $\mathbf{P}$. The number $r$ of POMs is chosen based on a prescription, in this case such that:

$$
\frac{\sum_{i=1}^{r} \lambda_{i}}{\sum_{i=1}^{n} \lambda_{i}} \geq 0.9999
$$

where $\lambda_{i}$ is the $i$ th ${ }^{i} \overline{\mathrm{POV}}$. In other words, assuming that the POVs are sorted in descending order, the first $r$ POMs that account for $99.99 \%$ of the total energy are considered.

\section{Correlation dimension and Lyapunov exponents}

Due to the sensitivity to initial conditions, the brute analysis of time series is obviously not a suitable means of characterising chaotic signals. Indicators such as the correlation dimension or the Lyapunov exponents are helpful in this case. Analysis of chaotic processes is often performed by reconstructing the phase space using the method of delays [21]. Consider $\mathrm{x}(\mathrm{t})$ as a single scalar component of a process of unknown dimension. The embedding procedure aims at generating a vector time series. This is achieved by choosing a time delay $\tau$ and an embedding dimension $m$ such that:

$$
\mathbf{y}(t)=[x(t) x(t+\tau) \ldots x(t+(m-1) \tau)]^{T}
$$

Part of the phase space is thus reconstructed from the time series $x(t)$ without even knowing the state space itself. The time delay $\tau$ and the embedding dimension $m$ are determined using the average mutual information [22] and the false nearest neighbours techniques respectively [23].

A possible measure of the spatial organisation of a chaotic attractor is given by the correlation integral [24]:

$$
C(r)=\lim _{n \rightarrow \infty} \frac{1}{n^{2}} \sum_{i, j=1}^{n} \theta(r-\|\mathbf{y}(i)-\mathbf{y}(j)\|)
$$

where the Heaviside function $\theta(\cdot)$ is equal to one when its argument is positive and zero when its argument is negative. The expression counts the number of points out of the data set within an hypersphere of radius $r$ and then divides by the square of the total number of points. As $r$ becomes small, the correlation dimension $D_{2}$ is found to be characterised by:

$$
C(r) \approx r^{D_{2}}
$$

and is thus given by the slope of a log-log plot of the correlation integral $C(r)$ vs. the distances $r$ :

$$
D_{2}=\frac{\partial \log [C(r)]}{\partial \log (r)}
$$

The Lyapunov exponents quantify the average rate of convergence or divergence of nearby trajectories. A positive exponent is an indication of the global instability and sensitivity to initial conditions and any system containing at least one positive exponent is defined to be chaotic. The magnitude of the exponent reflects the time scale on which system dynamics become unpredictable. The Lyapunov spectrum is the set of numbers describing the rate of exponential deformation of an infinitesimal hypersphere in phase space over time. Due to the contracting or expanding nature of directions in phase space, the sphere is deformed into an ellipsoid with principal axis $p_{i}(t)$. The $i$ th Lyapunov coefficient $\lambda_{i}$ is then defined by:

$$
\lambda_{i}=\lim _{t \rightarrow \infty} \frac{1}{t} \log _{2} \frac{p_{i}(t)}{p_{i}(0)}
$$

The approach proposed by Wolf [28] can be used to estimate non-negative Lyapunov exponents from a 
reconstructed attractor. The discussion here is limited to the estimation of the largest Lyapunov exponent. The evolution of a point and its nearest neighbour, initially distant from $L_{i}$, is monitored. The points evolve with time on different trajectories and once the distance $L^{\prime}$ between both points exceeds some threshold, the procedure is restarted by considering a new pair of points. This is repeated until the trajectory has traversed the entire data file. The largest Lyapunov exponent is then computed from:

$$
\lambda_{1}=\frac{1}{t_{n}-t_{0}} \sum_{i=1}^{M} \log _{2} \frac{L^{\prime}}{L_{i}}
$$

where $M$ is the number of times the procedure is restarted.

\section{Numerical application}

The numerical application under investigation is a clamped beam $(l=0.545 \mathrm{~m}$ and $E I=$ $49.3410^{-3} \mathrm{Nm}^{2}$ ) under the action of a sinusoidal force applied to the beam as shown in Fig. 1. Magnets are placed to the left and right of the free end to buckle the beam. The magnetic field creates forces which are assumed to be concentrated at the free end of the beam. This gives rise to a two-well potential $V(x)=$ $K_{1} x^{2} / 2+K_{2} x^{4} / 4$ where $K_{1}=-11.29 N / m$ and $K_{2}=2.7410^{4} \mathrm{~N} / \mathrm{m}^{3}$ are the magnet stiffness terms. The damping matrix is proportional to the stiffness matrix, i.e., $\mathbf{C}=1.310^{-3} \mathbf{K}$. The beam is discretised with seven beam elements (translational and rotational degrees of freedom at each node). In the literature, numerous works were realised on the single degree of freedom oscillator (e.g., references [25, 26]) but only a few studies tackled the multi degrees of freedom problem [27].

The system is simulated with a forcing frequency equal to $5 \mathrm{~Hz}$ and for amplitudes $F_{0}$ going from 0 up to $3.5 \mathrm{~N}$. To illustrate the varied dynamics of the system, a bifurcation diagram is presented in Fig. 2. This plot represents the horizontal displacement at the end of the beam (at the moment when the sine force is equal to $F_{0}$ ) as a function of the amplitude $F_{0}$. For a particular amplitude, the number of points is related to the period. The initial conditions at each amplitude are equal to zero and the bifurcation diagram is obtained by checking for any periodicity within the first $800 \mathrm{~s}$. In the absence of periodicity, the transients are not yet died out or the dynamics is non-periodic. It is worth pointing out that the beam can oscillate equally around either a magnet or the other depending on the

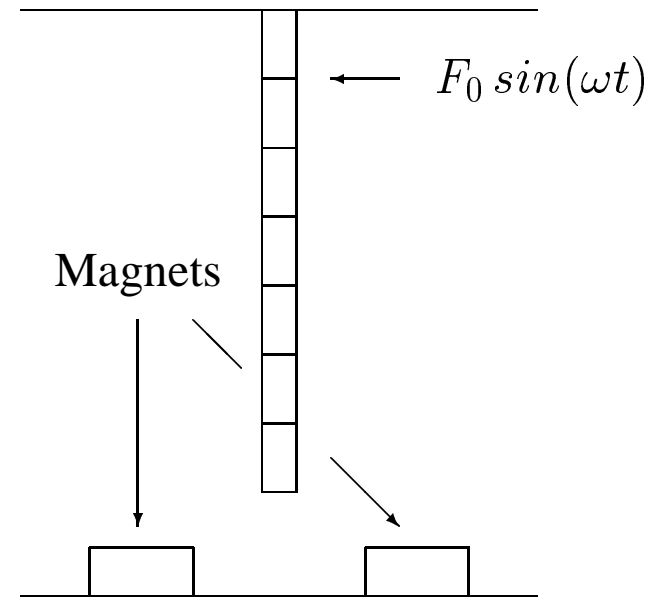

Figure 1: Clamped beam with two permanent magnets.

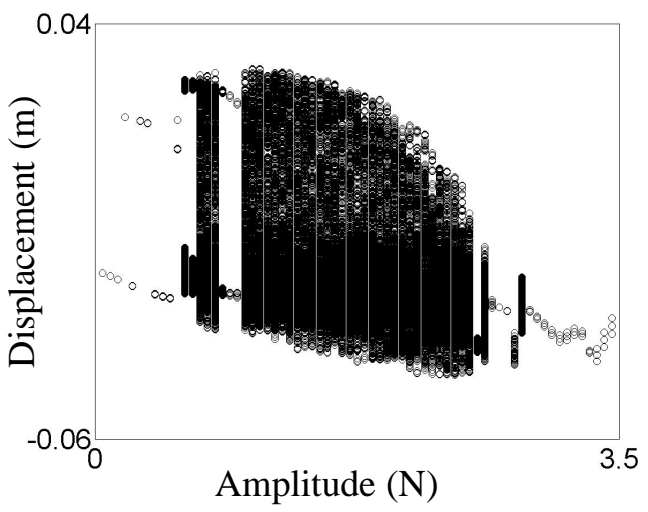

Figure 2: Bifurcation diagram of the full model.

initial conditions. This is the reason why points in the diagram may be associated with a positive or negative displacement. For some amplitudes, particularly between 1 and $2.5 \mathrm{~N}$, the number of points is large which may be interpreted as a non-periodic oscillation within the time allowed for the simulation. Fig. 3 shows phase portraits of the translational degree of freedom at the end of the beam for various amplitudes. For amplitudes equal to $0.1 \mathrm{~N}$ and $3.5 \mathrm{~N}$, the orbit is periodic while for the two selected intermediate amplitudes, i.e., $0.8 \mathrm{~N}$ and $2 \mathrm{~N}$, the orbits appear to be chaotic. This is confirmed by the greatest Lyapunov exponents equal to 2.85 and 3.03 respectively (Table 1).

As already mentioned, the POMs obtained from a chaotic orbit are to be used to build a low-dimensional model of the beam. However, the system is chaotic for a wide range of amplitudes, and it remains to determine the value of the amplitude for which the 

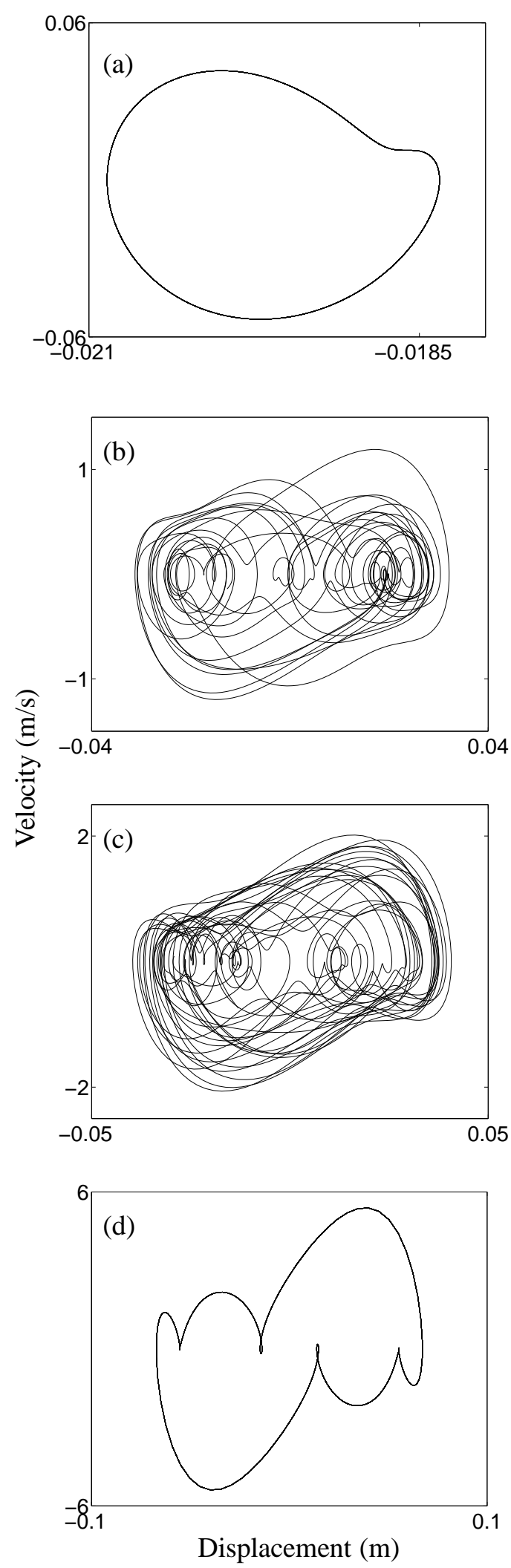

Figure 3: Phase portraits of the end of the beam computed from the full model. (a) $0.1 \mathrm{~N}$; (b) $0.8 \mathrm{~N}$; (c) 2 $\mathrm{N}$; (d) $3.5 \mathrm{~N}$.
Table 1: Greatest Lyapunov exponent for the full and reduced-order models.

\begin{tabular}{cccc}
\hline & $\begin{array}{c}\text { Full } \\
\text { model }\end{array}$ & $\begin{array}{c}\text { Five-mode } \\
\text { model }\end{array}$ & $\begin{array}{c}\text { Three-mode } \\
\text { model }\end{array}$ \\
\hline $0.8 \mathrm{~N}$ & 2.85 & 2.76 & $10^{-13}$ \\
$2 \mathrm{~N}$ & 3.03 & 2.95 & 0.71 \\
\hline
\end{tabular}

Table 2: Correlation dimension for the full and reduced-order models.

\begin{tabular}{cccc}
\hline & $\begin{array}{c}\text { Full } \\
\text { model }\end{array}$ & $\begin{array}{c}\text { Five-mode } \\
\text { model }\end{array}$ & $\begin{array}{c}\text { Three-mode } \\
\text { model }\end{array}$ \\
\hline $0.8 \mathrm{~N}$ & 2.65 & 2.52 & - \\
$2 \mathrm{~N}$ & 2.85 & 2.73 & 1.93 \\
\hline
\end{tabular}

modes are extracted. Interestingly enough, it was observed that the POMs do not vary significantly as long as the response is chaotic. The first five modes corresponding to an amplitude $F_{0}=1 \mathrm{~N}$ constitute 99.99 $\%$ of the total signal energy, and were thus chosen. These modes are displayed in Fig. 4 (see Table 3 for the POVs). All the degrees of freedom are used in the POD but only the translational degrees of freedom are illustrated in this figure. The greatest Lyapunov exponent computed for this amplitude is equal to 2.59, which confirms the chaotic nature of the response.

Fig. 5 depicts the phase portraits computed from the reduced-order model and is to be compared with Fig. 3. A convincing correspondence between both figures is obtained but the chaotic orbits, namely the orbits for the $0.8 \mathrm{~N}$ and $2 \mathrm{~N}$ levels, need further verification. The greatest Lyapunov exponent and the correlation dimension were thus computed and are listed in Table 1 and 2. It can clearly be seen that both indicators for the reduced-order model are in good concordance with the ones computed from the "full" model, i.e., the model discretised by the finite element modelling (14 degrees of freedom). Another possible means of assessing the results is to compare the occurrence of bifurcations. Indeed, bifurcations tend to be sensitive to modelling errors. To this end, the bifurcation diagram of the reduced-order model is presented in Fig. 6(a) and is in satisfactory agreement with the diagram obtained from the full model.

To confirm that the POMs extracted from a chaotic orbit represents a convenient choice, the results are also compared to another reduced-order model based on the POMs of a non-chaotic orbit, i.e., for $F_{0}=0.01$ $\mathrm{N}$ (see Fig. 7 and Table 3). In this case, only three 
Table 3: Normalised POVs for $0.01 \mathrm{~N}$ (top row) and $1 \mathrm{~N}$ (bottom row) levels.

\begin{tabular}{ccccc}
\hline 1st & 2nd & 3rd & 4th & 5th \\
\hline 0.9980 & 0.0014 & 0.0005 & $710^{-7}$ & $310^{-11}$ \\
0.6970 & 0.2702 & 0.0295 & 0.0031 & 0.0002 \\
\hline
\end{tabular}
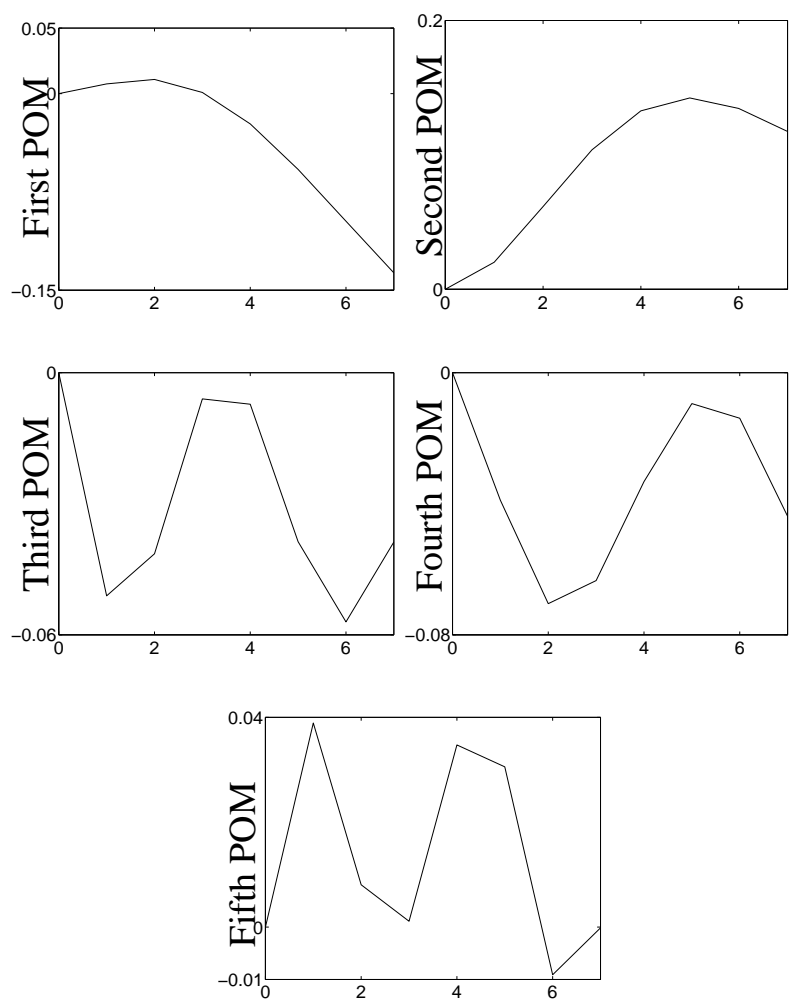

Displacement co-ordinate

Figure 4: POMs of the $1 \mathrm{~N}$ level.

POMs are retained in the model in accordance with the $99.99 \%$ criterion. However, it is worth noticing that five of these POMs were also used for reducedorder modelling, and that the results were not significantly improved. Fig. 8 displays the phase portraits computed from the three-mode model and is to be compared with Figs. 3, and 5. Severe distortions are now introduced in the phase portraits in comparison with the previous results. The orbit predicted for $F_{0}=0.8 \mathrm{~N}$ [Fig. $\left.8(\mathrm{~b})\right]$ is even periodic while the actual orbit is chaotic. The bifurcation diagram represented in Fig. 6(b), the greatest Lyapunov exponent and the correlation dimension listed in Tables 1 and 2 corroborate these observations.
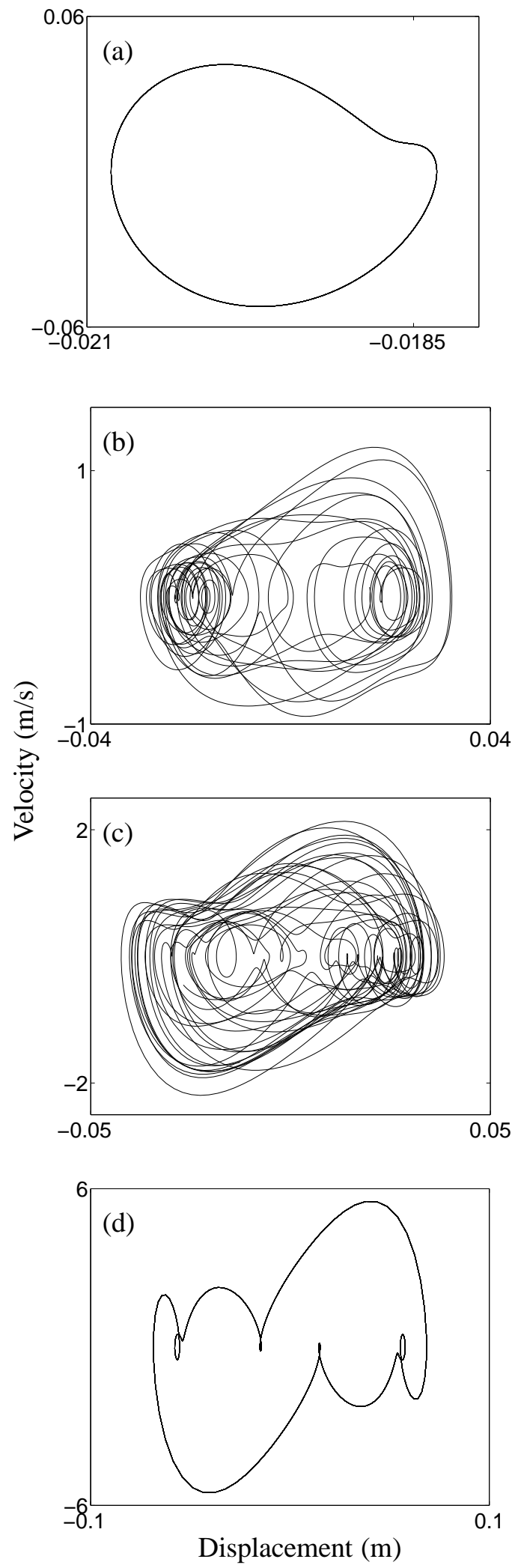

Figure 5: Phase portraits of the end of the beam computed from the five-mode model. (a) $0.1 \mathrm{~N}$; (b) 0.8 $\mathrm{N}$;(c) $2 \mathrm{~N}$; (d) $3.5 \mathrm{~N}$. 


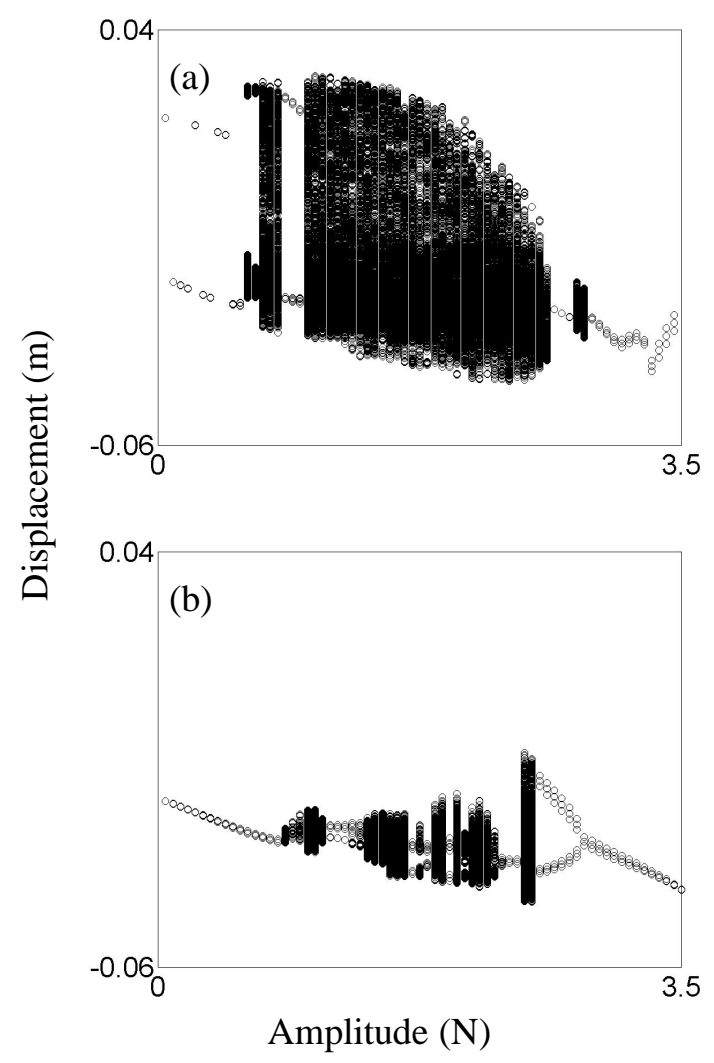

Figure 6: Bifurcation diagram of the reduced-order models. (a) Five-mode model; (b) three-mode model.
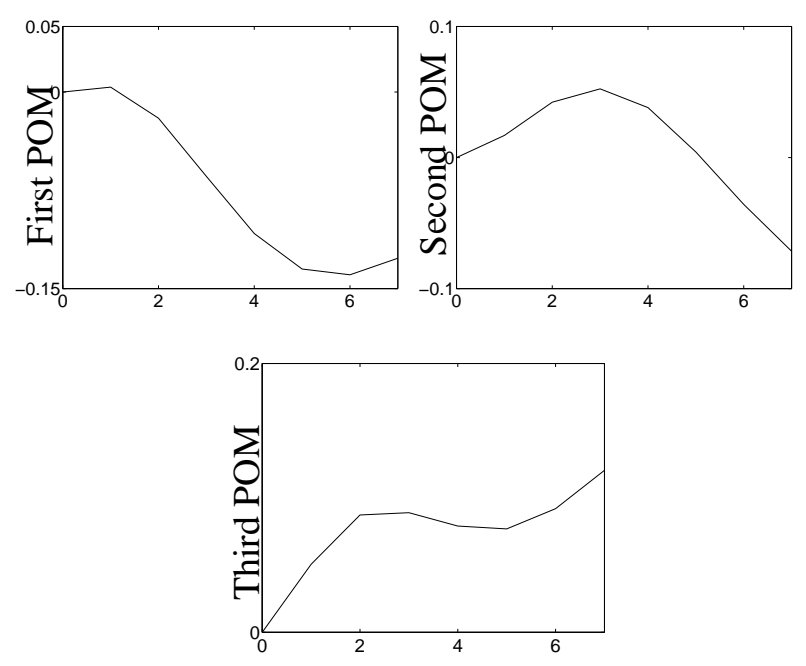

Displacement co-ordinate

Figure 7: POMs of the $0.01 \mathrm{~N}$ level.
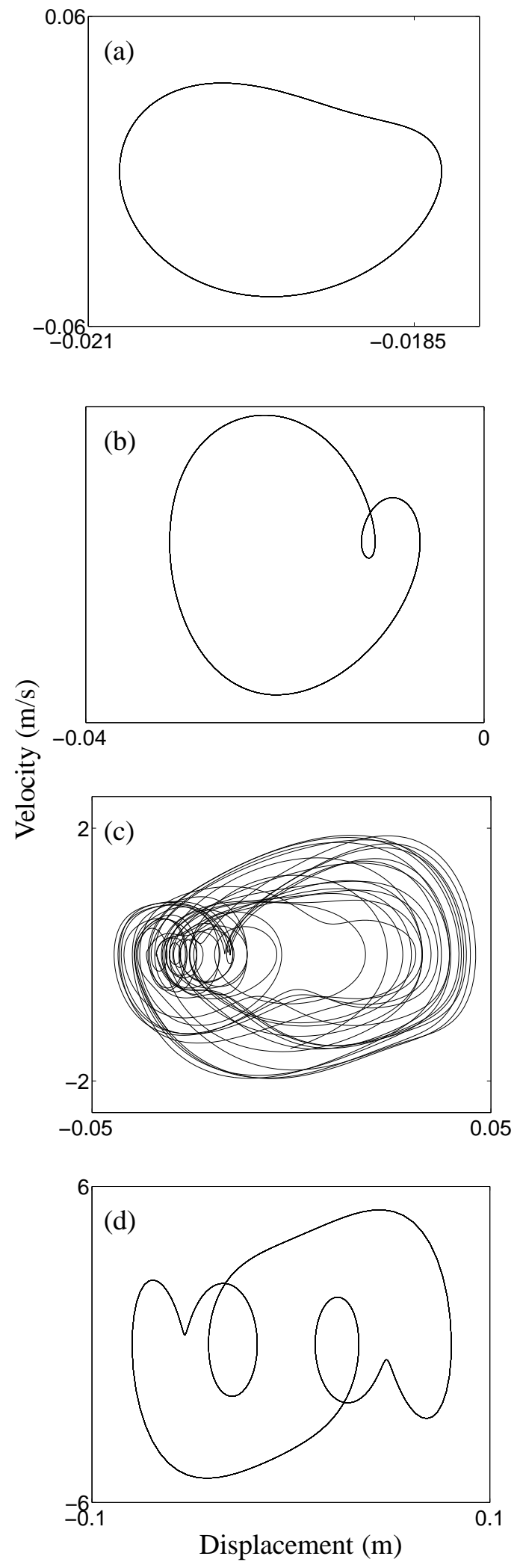

Figure 8: Phase portraits of the end of the beam computed from the three-mode model. (a) $0.1 \mathrm{~N}$; (b) 0.8 $\mathrm{N}$; (c) $2 \mathrm{~N}$; (d) $3.5 \mathrm{~N}$. 


\section{Conclusions}

In this paper, non-linear systems have been analysed through projection of the equations of motion onto the modes obtained from the POD instead of the modes shapes of the linearised system. The POMs of a chaotic orbit have been considered since these modes better capture the system dynamics. Although it is difficult to draw firm conclusions from a single numerical application, appreciable results have been obtained with the reduced-order model built with these POMs. Furthermore, significant improvements have been brought in comparison with a model built with the modes of a non-chaotic orbit.

\section{Acknowledgements}

The author G. Kerschen is supported by a grant from the Belgian National Fund for Scientific Research (FNRS) which is gratefully acknowledged. The author B. Feeny is supported by the National Science Foundation (CMS-0099603).

\section{References}

[1] B.F. Feeny and R. Kappagantu, On the physical interpretation of proper orthogonal modes in vibrations, Journal of Sound and Vibration 211 (1998) 607-616.

[2] G. Kerschen and J.C. Golinval, Physical interpretation of the proper orthogonal modes using the singular value decomposition, Journal of Sound and Vibration 249 (2002) 849-865.

[3] M.F.A. AZEEZ and A.F. VAKAKIS 2001 Journal of Sound and Vibration 240, 859-889 . Proper orthogonal decomposition of a class of vibroimpact oscillations.

[4] R. Kappagantu and B.F. Feeny, An optimal modal reduction of a system with frictional excitation, Journal of Sound and Vibration 224 (1999) 863-877.

[5] K. Karhunen, Uber lineare methoden in der wahrscheinlichkeitsrechnung, Annals of Academic Science Fennicae, Series A1 Math. Phys. 37 (1946).

[6] D. Kosambi, Statistics in function space, Journal of Indian Mathematical Society 7 (1943) 76-88.
[7] M. Loeve, Fonctions aléaloires de second ordre, Compte Rend. Acad. Sci. (Paris 1945).

[8] M.A. Obukhov, Statistical description of continuous fields, T. Geophys. Int. Akad. Nauk. USSR 24 (1954) 3-42.

[9] V.S. Pougachev, General theory of the correlations of random functions, Izv. Akad. Nauk. USSR 17 (1953) 1401-1402.

[10] G. Berkooz, Observations on the Proper Orthogonal Decomposition in Studies in Turbulence (Springer-Verlag, New-York, 1992).

[11] K. Pearson, On lines and planes of closest fit to systems of points in space, Philosophical Magazine 2 (1901) 559-572.

[12] H. Hotelling, Analysis of a complex of statistical variables into principal components, Journal of Educational Psychology 24 (1933) 417-441.

[13] J.P. Cusumano, M.T. Sharkady, B.W. Kimble, Experimental measurements of dimensionality and spatial coherence in the dynamics of a flexible-beam impact oscillator, Philosophical Transactions of the Royal Society of London, 347 (1994) 421-438.

[14] E. Kreuzer, O. Kust, Proper orthogonal decomposition - an efficient means of controlling selfexcited vibrations of long torsional strings, Nonlinear Dynamics and Control ASME DE-Vol. 91 (1996) 105-110.

[15] M.F.A. Azeez, A.F. Vakakis, Numerical and experimental analysis of the nonlinear dynamics due to impacts of a continuous overhung rotor, Proceedings of ASME Design Engineering Technical Conference (Sacramento, CA, 1997).

[16] A. Benguedouar, Proper orthogonal decomposition in dynamical modeling: a qualitative dynamic approach, Ph.D. Thesis (Boston University, MA, 1995).

[17] P.M. Fitzsimons, C. Rui, Determining low dimensional models of distributed systems, Advances in Robust and Nonlinear Control Systems, ASME DSC 53 (1993).

[18] I.T. Georgiou, I.B. Schwartz, Dynamics of large scale coupled structural-mechanical systems: a 
singular perturbation proper orthogonal decomposition approach, SIAM Journal of Applied Mathematics 59 (1999) 1178-1207.

[19] V. Lenaerts, G. Kerschen, J.C. Golinval, Proper orthogonal decomposition for model updating of non-linear mechanical systems, Mechanical Systems and Signal Processing 15 (2001) 31-43.

[20] P. Holmes, J.L. Lumley, G. Berkooz, Turbulence, Coherent Structures, Dynamical Systems and Symmetry (Cambridge, New York, 1996).

[21] F. Takens, Detecting strange attractors in turbulence. In Rand, D. A. and Young, L.-S., editors, Dynamical Systems and Turbulence: Lecture Notes in Mathematics, Vol. 898, 336-381. (Springer-Verlag, Berlin, 1981).

[22] A.M. Fraser, H.L. Swinney, Independant coordinates for strange attractors from mutual information, Physical Review A 33 (1986) 11341140 .

[23] M.B. Kennel, R. Brown, H.D.I. Abarbanel, Determining embedding dimension for phasespace reconstruction using a geometrical construction, Physical Review A 45 (1992) 34033411.

[24] P. Grassberger, I. Procaccia, Measuring the strangeness of strange attractors, Physica D 9 (1983) 189-208.

[25] P. Holmes, A nonlinear oscillator with a strange attractor, Phil. Trans. of the Royal Society of London 292 (1979) 419-448.

[26] F.C. Moon, Experiments on chaotic motions of a forced nonlinear oscillator: strange attractors, Journal of Applied Mechanics 47 (1980) 190196.

[27] D.M. Tang, E.H. Dowell, On the threshold force for chaotic motions for a forced buckled beam, Journal of Applied Mechanics 55 (1988) 190196.

[28] A. Wolf, J.B. Swift, H.L. Swinney, J.A. Vastano, Determining Lyapunov exponents from a time series, Physica D 16 (1985) 285-317. 